\title{
Response of a Maize Synthetic to Selection for Components of Partial Resistance to Exserohilum turcicum
}

\author{
M. L. Carson, USDA-ARS, Cereal Disease Laboratory, St. Paul, MN 55108; formerly USDA-ARS, Plant Science \\ Research, Raleigh, NC 27695
}

\begin{abstract}
Carson, M. L. 2006. Response of a maize synthetic to selection for components of partial resistance to Exserohilum turcicum. Plant Dis. 90:910-914.

A synthetic population of maize (Zea mays) was created from five inbred lines of varying levels of partial resistance to northern leaf blight (NLB). This synthetic was subjected to three cycles of recurrent phenotypic selection with pollen control for either increased latent period or decreased lesion length. A selection intensity of ca. $10 \%$ was used in each selection cycle. The original synthetic and three advanced selection cycles for each of the two components of partial resistance were evaluated in field trials in the summers of 1999 and 2001, and in greenhouse trials. Selection for increased latent period was more effective in improving resistance to NLB (20 to $27 \%$ gain/cycle) (as measured by area under the disease progress curve [AUDPC]) than was selection for decreased lesion length (14 to 18\% gain/cycle). Responses in AUDPC to selection for either component of resistance were linear in the 1999 field trial, but were quadratic (decreased response in advanced cycles) in the 2001 trial. Selection for increased latent period in the field resulted in a 0.6-day increase in latent period per selection cycle when measured in the greenhouse and a 2-day increase per selection cycle when measured in the field. Selection for decreased lesion length in the field did not significantly alter latent period in the greenhouse. These results support using selection for increased latent period as an effective means of improving partial resistance to NLB in maize populations. Decreased lesion length was more difficult to measure and selection based on this criterion was less effective in improving partial resistance.
\end{abstract}

Additional keywords: Helminthosporium turcicum, Setosphaeria turcica

Northern leaf blight (NLB), caused by the fungus Exserohilum turcicum (Pass.) Leonard \& Suggs (teleomorph $=\mathrm{Se}$ tosphaeria turcica Leonard \& Suggs; syn. = Helminthosporium turcicum), is an important foliar disease of maize (Zea mays L.) that occurs worldwide virtually everywhere maize is grown $(13,32,41)$. The disease is most prevalent and damaging when cool to moderate temperatures and moist conditions prevail during the growing season $(13,32,41)$. E. turcicum overwinters in crop residues and initiates epidemics with conidia produced on those residues (27).

The disease can cause extensive defoliation during the grain-filling period, resulting in grain yield losses of $50 \%$ or more $(13,23,24,37)$. Resistance in maize to NLB is generally classified as one of two types: major gene resistance, conferred by the $\mathrm{Ht}$, Ht2, Ht3, or HtN genes, that is racespecific, and partial resistance that is under

Corresponding author: M. L. Carson

E-mail: mcarson@umn.edu

Accepted for publication 22 February 2006.

DOI: 10.1094/PD-90-0910

This article is in the public domain and not copyrightable. It may be freely reprinted with customary crediting of the source. The American Phytopathological Society, 2006. polygenic control and is effective against all pathogen biotypes (11-16,22,25,35, 36,38). The Ht1, Ht2, and Ht3 genes confer a "chlorotic lesion" type of reaction to the pathogen $(12,14,15)$, whereas the $H t N$ gene results in a delay of symptoms until after anthesis $(11,26)$. Virulence to each of these genes has been demonstrated in the population of E. turcicum in the United States and elsewhere (20,21,30,33,34, 39,43). An additional recessive gene, $h t 4$, that confers a "chlorotic halo" response to infection by $E$. turcicum has been described, but its effectiveness in controlling NLB is not clear (6). Emphasis in commercial maize breeding programs in the United States has been on exploiting partial resistance, although the Htl gene was used extensively prior to the discovery of isolates of E. turcicum with corresponding virulence to it.

Partial resistance of maize to NLB is inherited in a polygenic manner with mostly additive gene action involved $(16,18)$. Mapping studies using reciprocal translocations and molecular markers indicate that quantitative trait loci (QTL) on all 10 chromosomes of maize may be involved $(2,8-10,17,28,40)$. Partial resistance of maize to NLB is expressed as a reduction in development of the disease and percent leaf area infected, which in turn may result from expression of several components, including incubation period, latent period, lesion size, lesion numbers, lesion expansion rate, and sporulation intensity $(1,2,5,7,25,29,31)$. An increase in latent or incubation period is the one component most related to disease development on adult plants and is expressed over a range of temperature and light conditions $(1,7,31)$. Several studies have attempted to map QTL for specific components of partial resistance to NLB. QTL for increased latent (or incubation) period for NLB in the commercially important inbred line Mo17 often mapped to the same chromosome arms as loci governing percent disease severity or area under the disease progress curve (AUDPC) on adult plants $(2,8,38)$. A similar correlation was found in mapping NLB resistance QTL in the tropical inbred line CML202 and in an early maturing European flint $x$ dent population $(28,40)$. QTL for reduced lesion numbers also mapped to many of the same locations as reduced NLB severity, and lesion numbers were correlated with NLB severity $(2,10)$. Other possible components of NLB resistance such as lesion size and sporulation intensity are only weakly associated with disease severity and associated QTL $(2,10)$.

The objective of this research was to determine the efficacy of selection for two components of partial resistance, increased latent period and shorter lesion length, on improving the NLB resistance in a maize synthetic.

\section{MATERIALS AND METHODS}

Creation of the maize synthetic. A synthetic population was created by intercrossing five maize inbred lines varying in level of partial resistance: 69-1, Mo17, B37, A619, and A632. These inbred lines are recessive at all of the major $\mathrm{Ht}$ gene loci. An experimental line, 69-1, was derived from the maize synthetic BS-19 and has a very high level of partial resistance expressed as an extended latent period $(4,5)$. Mo17 is an inbred line with an acceptable level of partial resistance and has been used in QTL mapping studies of NLB resistance (2,8-10). B37 has a low level of partial resistance to NLB. A619 and A632 are considered very susceptible to NLB. The latter four inbred lines are all public releases that were widely used in the hybrid seed industry and are still represented in the pedigrees of inbred lines currently used in commercial hybrids. The five parental lines were crossed in a half-diallel 
(all possible F1 crosses without reciprocals) manner. The resulting F1s were also crossed in a half-diallel manner, and the resulting double crosses were allowed to random mate in isolation to form the initial population (Cycle 0 ).

Selection for components of partial resistance. The base population was subjected to selection for two separate components of partial resistance to NLB: increased latent period and decreased lesion length. Selection was practiced in field plots consisting of at least 20 rows of the population(s), $6 \mathrm{~m}$ long and spaced $0.9 \mathrm{~m}$ apart. Rows were seeded with 25 seeds each and were not thinned. Plants were inoculated at the four- to six-leaf stage by placing ca. 20 to 30 seeds of a sorghum grain culture of a virulent isolate of $E$. turcicum (Et10) into the leaf whorl. Et10 is a race $\mathrm{O}$ isolate originally collected in 1985 from Wilkes County, NC. The sorghum grain inoculum was produced by soaking sorghum seed overnight in water, draining off the excess, and placing the moistened seed into flasks and autoclaving them for $1 \mathrm{~h}$. Flasks of autoclaved, moistened sorghum seed were inoculated with conidial suspensions prepared by washing conidia from 10-day-old lactose caseinate agar (LCA) cultures of E. turcicum. The inoculated sorghum cultures were grown for 2 weeks at room temperature and then stored at $4^{\circ} \mathrm{C}$. Latent period was measured as the time (in days) from inoculation to the appearance of the first NLB lesion on a plant. Plants were removed as they became symptomatic until ca. $10 \%$ of the plants remained. These remaining plants were intermated by bulking pollen from these selected plants and using it to pollinate individual plants. This intermating was done on two separate dates to accommodate the range in plant maturities within the populations. Equal amounts of seed from each pollinated ear were bulked to form the next selection cycle. Lesion length was measured as the length $(\mathrm{cm})$ of a single NLB lesion 5 days after its initial appearance. A single lesion on individual plants was marked and dated using an indelible marking pen on the infected leaf on the day the lesion first began to expand from an infection point. Lesion lengths were measured and written on the same leaves 5 days later, and the plant was marked with high-visibility spray paint to indicate that lesion length data for that plant had been recorded. When data from all plants in the population had been recorded, the $10 \%$ of plants with shortest lesions were intermated as described above to form the next selection cycle. After the initial selection in the original synthetic (Cycle 0), further selection cycles for the two components of resistance were kept separate, forming two separate lines of selection. Two additional selection cycles were conducted for the two traits, using the methodology outlined above.
Field evaluations of selected populations. Field trials of the original synthetic (Cycle 0) and advanced cycles selected for increased latent period (LLP Cycle 1, LLP Cycle 2, and LLP Cycle 3) and for decreased lesion length (SLL Cycle 1, SLL Cycle 2, and SLL Cycle 3) were conducted at the Central Crops Research Station near Clayton, NC, during the summers of 1999 and 2001. In addition to the seven populations above, the NLB-susceptible hybrid A632 × A619 was included as a control treatment. The experiments consisted of randomized complete blocks with treatment units consisting of single rows $6 \mathrm{~m}$ long and $0.9 \mathrm{~m}$ apart, planted with 25 seeds per plot. There were 10 replications planted in 1999 on 14 April and seven in 2001 on 10 April. No trial was planted in 2000, as a seed storage failure seriously reduced seed viability. Remnant seed of the populations was planted, and the populations were regenerated for the 2001 trial by random plant-to-plant crossing (sib mating) with each plant used as either a male or female in nurseries in the summer of 2000 and in a winter nursery in southern Florida in 2000-2001. Field trials were inoculated with E. turcicum as described above. Whole plots were visually rated for NLB severity (percent leaf area diseased) four times during the grain-filling period, and area under the disease progress curve was calculated (42). In addition to collecting NLB severity data, the date when $50 \%$ of plants within a plot were symptomatic was recorded in the 1999 trial as a measure of latent period. No latent period data were recorded in 2001 due to poor and inconsistent initial infection by E. turcicum in the plots.

Greenhouse trials of selected populations. Three greenhouse trials were conducted in January and February 2002 to measure latent periods of the original synthetic and the advanced selection cycles, as well as the check hybrid A632 × A619. Each trial was a randomized complete block design with seven replications.
Seeds were planted 10 per row in flats containing a commercial artificial potting medium (MetroMix 200, W. R. Grace, Inc.), with four rows per flat, two flats per block. Each block consisted of a single row each of the original synthetic (Cycle 0 ), advanced cycles for each of the two selection schemes, and the check hybrid, for a total of eight treatments. Seedlings were not thinned, so the final number of plants per row varied. Flats were fertilized twice weekly with a solution of a commercial soluble (20-20-20 NPK) fertilizer (Peter's Professional Plant Food; W. R. Grace, Inc.).

Plants were inoculated 3 weeks after planting. Approximately $0.1 \mathrm{ml}$ of a $10^{4}$ conidia/ml suspension of E. turcicum (a mixture of 11 isolates of race $\mathrm{O}$ ) was pipetted into the leaf whorl, and flats were placed overnight (16 h) in a mist chamber. Inoculum was prepared by washing conidia from the surface of LCA cultures of the E. turcicum isolates, suspending in tap water to which Tween $20(1 \mathrm{drop} / 100 \mathrm{ml})$ had been added, and the resulting suspension adjusted to final concentration with the aid of a hemacytometer.

Beginning 7 days after inoculation, plants were inspected daily for the presence of necrotic lesions, and the number of symptomatic plants in each row was recorded. The latent period of individual plants was recorded as the number of days from inoculation to necrotic lesion formation.

Data analysis. Data were subjected to analysis of variance using SAS GLM, and the variation among selection cycles for each component of resistance was partitioned into the linear and quadratic responses to selection by regressing NLB disease reaction data on selection cycle number. The selection response per cycle was calculated as the slope of the linear regression. The treatments significantly interacted with years in the field experiments, so selection responses were estimated separately for each year.

Table 1. Reactions of a maize synthetic and advanced selection cycles to phenotypic recurrent selection for components of partial resistance to northern leaf blight caused by Exserohilum turcicum

\begin{tabular}{lccc}
\hline Population & $\begin{array}{c}\text { AUDPC } \\
\text { (percent-days) }\end{array}$ & $\begin{array}{c}\text { Latent period- } \\
\text { greenhouse (days) }\end{array}$ & $\begin{array}{c}\text { Latent period }^{\mathbf{b}} \text { - } \\
\text { field, 1999 }\end{array}$ \\
\hline A632 $\times$ A619 (check) & 816 & 11.4 & 0.6 \\
Cycle 0 & 787 & 13.6 & 1.5 \\
LLP Cycle 1 & 563 & 13.8 & 3.6 \\
LLP Cycle 2 & 376 & 14.9 & 5.7 \\
LLP Cycle 3 & 282 & 15.2 & 7.4 \\
SLL Cycle 1 & 647 & 12.7 & 2.3 \\
SLL Cycle 2 & 527 & 12.7 & 4.4 \\
SLL Cycle 3 & 406 & 13.0 & 4.7 \\
FLSD $(P<0.05)$ & 197 & & \\
\hline
\end{tabular}

a AUDPC $=$ area under the disease progress curve.

${ }^{b}$ Latent period in the field was measured as days past 1 June on which $50 \%$ of plants were symptomatic.

${ }^{\mathrm{c}} \mathrm{LLP}=$ advanced cycles selected for increased latent period.

${ }^{\mathrm{d}} \mathrm{SLL}=$ advanced cycles selected for shorter lesion lengths.

e Fisher's least significant difference. 


\section{RESULTS}

Field trials. Development of NLB was greater in the 1999 than the 2001 field trial. NLB severity on the susceptible check hybrid reached a high of $92 \%$ by the last rating date near physiological maturity in 1999 , but only reached $43 \%$ at the same growth stage in 2001. For reasons that were not readily apparent, some plants escaped initial infection after inoculation in 2001, so disease development was delayed compared with 1999. Final NLB severities on the populations ranged from 54 to $85 \%$ and 8 to $43 \%$ in 1999 and 2001, respectively. The date when $50 \%$ of plants were symptomatic (an indirect measure of latent period) in the 1999 field trial ranged from 1 June $($ A632 $\times$ A619) to 7 June (LLP Cycle 3; Table 1). In greenhouse trials, mean latent periods (days to first necrotic lesion) ranged from 11.4 days for the check hybrid A632 × A619 to 15.2 days for the third cycle of selection for long latent period (LLP Cycle 3; Table 1).

Direct selection for increased latent period resulted in a linear decrease in AUDPC of 202 percent-days (a 20\% reduction) per cycle in the 1999 field trial (Fig. 1A). In the 2001 field trial, the response in AUDPC to selection for increased latent period was quadratic, with most of the improvement occurring in the first two selection cycles (Fig. 2A). The linear response to selection averaged 120 percent-days or $27 \%$ per cycle in 2001 . Selection for decreased lesion length resulted in an average decrease in AUDPC of 149 percent-days or $14 \%$ per cycle in
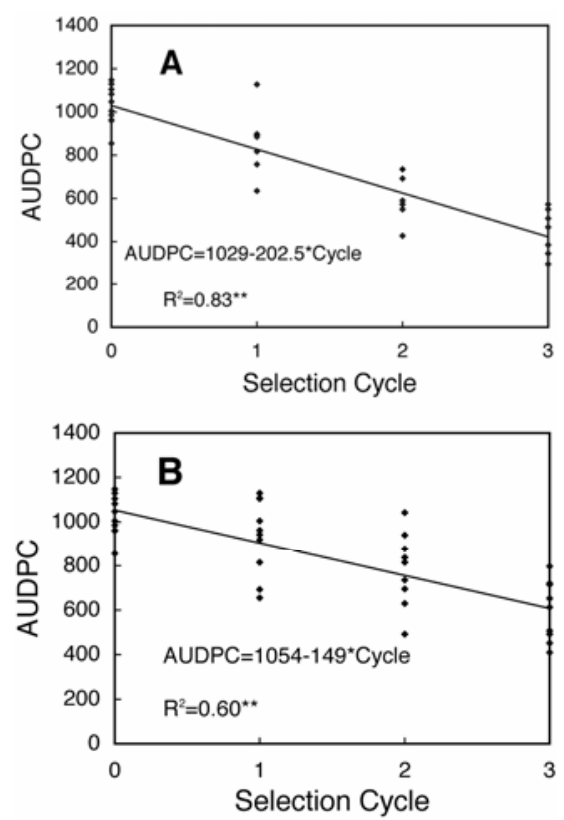

Fig. 1. Response in area under the disease progress curve (AUDPC) of northern leaf blight to phenotypic recurrent selection for increased latent period (A) or decreased lesion length (B) in a maize synthetic. Data are from a field experiment conducted at the Central Crops Research Station in Clayton, NC, in 1999.
1999 , and 80.4 percent-days or $18 \%$ in 2001 (Figs. 1B and 2B, respectively).

The direct response to selection for increased latent period resulted in an increase of 2 days per selection cycle when measured in 1999 field trials as the number of days after 1 June when $50 \%$ of plants were symptomatic (Fig. 3A). Selection for decreased lesion length produced a correlated response of a 1.2-day increase in the number of days after 1 June when $50 \%$ of plants were symptomatic per selection cycle in the 1999 field trial (Fig. 3B).

Greenhouse trials. Selection for increased latent period produced an average gain per cycle of 0.6 days or $\sim 4 \%$ when measured on seedling plants in greenhouse trials (Fig. 4A). Selection for reduced lesion length had no significant effect on latent period as measured on seedling plants in the greenhouse (Fig. 4B).

\section{DISCUSSION}

Selection for either increased latent period or decreased lesion length is an effective means of improving maize populations for partial resistance to NLB. Selection for either of these components of partial resistance is advantageous because it can be practiced before pollination, thereby allowing the selection of both female and male parents. Theoretically, this doubles the selection efficiency compared with simple
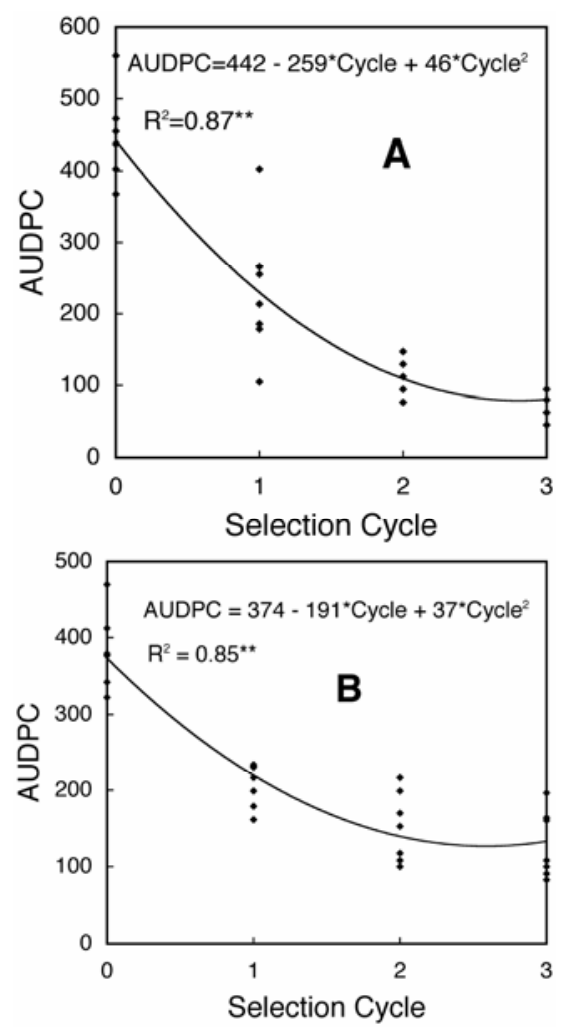

Fig. 2. Response in area under the disease progress curve (AUDPC) of northern leaf blight to phenotypic recurrent selection for increased latent period (A) or decreased lesion length (B) in a maize synthetic. Data are from a field experiment conducted at the Central Crops $\mathrm{Re}$ search Station in Clayton, NC, in 2001. mass selection where selection is practiced on open-pollinated female plants at some time after pollination. In this study, selection for increased latent period resulted in an average gain of 20 to $27 \%$ per cycle (as measured by reduction in AUDPC). These gains are nearly twice that reported by Campana and Pataky (3) in three of four sweet maize populations where phenotypic mass selection was conducted for reduced NLB severity. These gains are also somewhat greater than those reported by Jenkins et al. (19), whose average gain per cycle across nine populations varied from 11 to $21 \%$. In both these studies, selection was for reduced NLB severity before anthesis, and both male and female plants were selected for recombination. Because most NLB development occurs after anthesis, selection based on severity before anthesis may not be as effective as selection based on either a single NLB severity rating measured later in epidemic development or on multiple severity ratings taken during the NLB epidemic, such as AUDPC.

Selection for reduced lesion length was less effective in increasing resistance to NLB than was selection for increased latent period, when comparing average gain per selection cycle. Selection for reduced lesion length also proved more tedious to measure than latent period on plants in field plots, at least as it was measured in this study where lesions of a uniform age were measured. Based on previous studies where little relation was found between
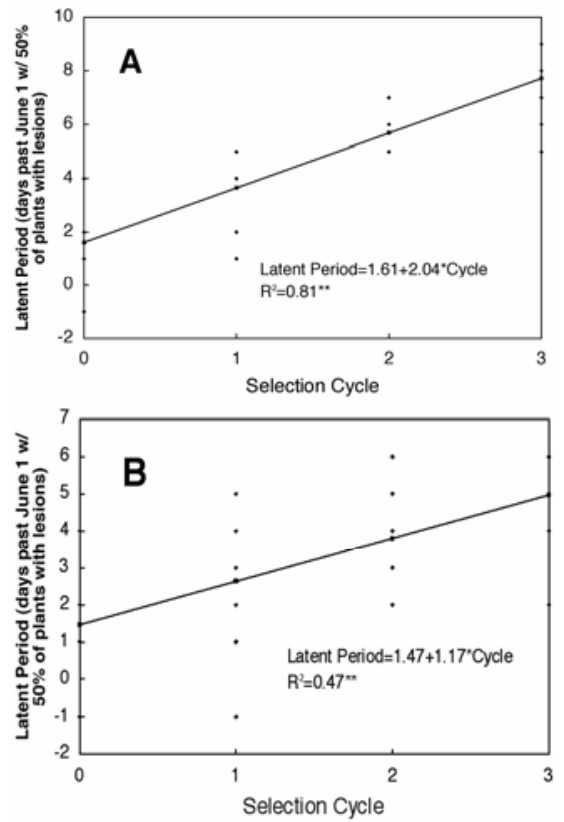

Fig. 3. Response in latent period (measured as the number of days past 1 June when $50 \%$ of plants were symptomatic) of northern leaf blight to phenotypic recurrent selection for increased latent period (A) or decreased lesion length (B) in a maize synthetic. Data are from a field experiment conducted at the Central Crops Research Station in Clayton, NC, in 1999. 
lesion size and NLB severity and lesion size itself was found to be of low heritability $(2,10)$, it is somewhat surprising at first glance that selection for smaller lesion size was effective in improving NLB resistance. In the previous studies, lesion size was measured on lesions of mixed and undetermined age, whereas in this study lesions were measured at a uniform age ( 5 days after their first appearance), and conceivably this would be subject to less extraneous error due variability in age of the lesion and as a consequence be of higher heritability.

The improvement in partial resistance to NLB seen in the 1999 field trial was mirrored by the increase in latent period in advanced cycles selected for either increased latent period or decreased lesion length. It is unfortunate that latent periods could not be reliably measured in the 2001 field trial, but it was evident from the lack of visible infection points (necrotic flecks) that many plants escaped initial infection.

The efficacy of selection for increased latent period was also evident in seedling greenhouse trials. The response, although statistically significant, was a modest $4 \%$ gain per cycle. There was no significant response of latent period to selection for shorter lesion length detected in greenhouse trials. A previous study has shown that although latent period on seedlings in the greenhouse is closely related to partial resistance seen under field conditions, differences in latent periods among maize
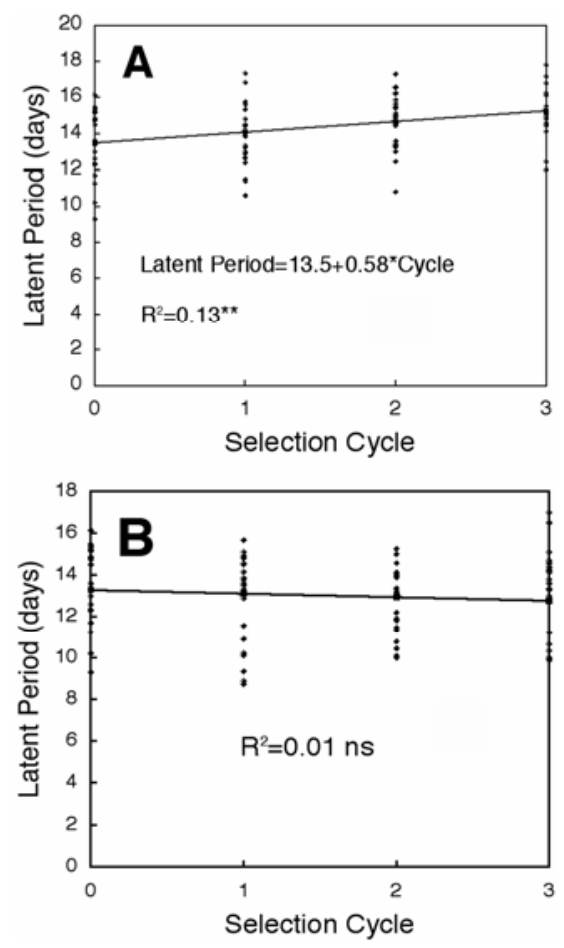

Fig. 4. Response in latent period (in days) of northern leaf blight to phenotypic recurrent selection for increased latent period (A) or decreased lesion length (B) in a maize synthetic. Data are from three runs of a greenhouse experiment. genotypes are smaller under greenhouse conditions than in the field (5).

Previous studies have documented the close relationship between latent period and partial resistance, but this study is the first to clearly demonstrate the efficacy of selection for increased latent period in improving partial resistance in maize to NLB. Selection for increased latent period was also clearly superior to selection for decreased lesion length in both efficacy and ease of use. Given the preponderance of evidence of the importance of latent period in partial resistance to NLB in diverse maize cultivars, it is tempting to generalize that latent period is universally the most important component of partial resistance in maize to NLB. However, it is entirely possible that other components of resistance may be most important in other maize germ plasm. The inbred lines used in the synthesis of the population that was the subject of selection in this study were specifically selected to represent a range of latent periods; therefore it is not particularly surprising that sufficient variability for latent period existed in the population and selection was very effective.

\section{LITERATURE CITED}

1. Adipala, E., Lipps, P. E., and Madden, L. V. 1993. Reaction of maize cultivars from Uganda to Exserohilum turcicum. Phytopathology 83:217-223.

2. Brewster, V. A., Carson, M. L., and Wicks, Z. W., III. 1992. Mapping components of partial resistance to northern leaf blight of maize using reciprocal translocations. Phytopathology 82:225-229.

3. Campana, A., and Pataky, J. K. 2005. Frequency of the $\mathrm{Htl}$ gene in populations of sweet corn selected for resistance to Exserohilum turcicum race 1. Phytopathology 95:85-91.

4. Carson, M. L. 1992. Diversity of types of resistance to northern leaf blight in the BS19 maize synthetic. (Abstr.) Phytopathology 82:991.

5. Carson, M. L. 1995. Inheritance of latent period length in maize infected with Exserohilum turcicum. Plant Dis. 79:581-585.

6. Carson, M. L. 1995. A new gene in maize conferring the "chlorotic halo" reaction to infection by Exserohilum turcicum. Plant Dis. 79:717-720.

7. Carson, M. L., and Van Dyke, C. G. 1994. Effect of light and temperature on expression of partial resistance of maize to Exserohilum turcicum. Plant Dis. 78:519-522.

8. Dingerdissen, A. L., Geiger, H. H., Lee, M., Schechert, A., and Welz, H. G. 1996. Interval mapping of genes for quantitative resistance of maize to Setosphaeria turcica, cause of northern leaf blight, in a tropical environment. Mol. Breed. 2:143-156.

9. Freymark, P. J., Lee, M., Woodman, W. L., and Martinson, C. A. 1993. Quantitative and qualitative trait loci affecting host-plant response to Exserohilum turcicum in maize (Zea mays L.). Theor. Appl. Genet. 87:537-544.

10. Freymark, P. J., Lee, M., Woodman, W. L., and Martinson, C. A. 1994. Molecular-markerfacilitated investigation of host-plant response to Exserohilum turcicum in maize (Zea mays L.): Components of resistance. Theor. Appl. Genet. 88:305-313.

11. Gevers, H. O. 1975. A new major gene for resistance to Helminthosporium turcicum leaf blight of maize. Plant Dis. Rep. 59:296-299.
12. Hooker, A. L. 1963. Inheritance of chloroticlesion resistance to Helminthosporium turcicum in seedling corn. Crop Sci. 3:660662.

13. Hooker, A. L. 1975. Helminthosporium turcicum as a pathogen of corn. Rep. Tottori Mycol. Inst. (Jpn.) 12:115-125.

14. Hooker, A. L. 1977. A second major gene locus in corn for chlorotic-lesion resistance to Helminthosporium turcicum. Crop Sci. 17:132135.

15. Hooker, A. L. 1981. Resistance to Helminthosporium turcicum from Tripsacum floridanum incorporated into corn. Maize Genet. Coop. News Lett. 55:87-88.

16. Hughes, G. R., and Hooker, A. L. 1971. Gene action conditioning resistance to northern corn leaf blight in maize. Crop Sci. 11:180183.

17. Jenkins, M. T., and Robert, A. L. 1961. Further genetic studies of resistance to Helminthosporium turcicum Pass. in maize by means of chromosomal translocations. Crop Sci. 1:450455.

18. Jenkins, M. T., Robert, A. L., and Findley, W. R., Jr. 1952. Inheritance of resistance to Helminthosporium leaf blight in populations of $\mathrm{F}_{3}$ progenies. Agron. J. 44:438-442.

19. Jenkins, M. T., Robert, A. L., and Findley, W. R. 1954. Recurrent selection as a method for concentrating genes for resistance to Helminthosporium leaf blight in corn. Agron. J. 46:89-94

20. Leonard, K. J., Levy, Y., and Smith, D. R. 1989. Proposed nomenclature for pathogenic races of Exserohilum turcicum on corn. Plant Dis. 73:776-777.

21. Pataky, J. K., Carson, M. L., and Mosely, P. R. 1991. Race 23N of Exserohilum turcicum in Florida. Plant Dis. 75:863.

22. Pataky, J. K., Perkins, J. M., and Leath, S. 1986. Effects of qualitative and quantitative resistance on the development and spread of northern leaf blight of maize caused by Exserohilum turcicum races 1 and 2. Phytopathology 76:1349-1352.

23. Perkins, J. M., and Pedersen, W. L. 1987. Disease development and yield losses associated with northern leaf blight on corn. Plant Dis. 71:940-943.

24. Raymundo, A. D., and Hooker, A. L. 1981 Measuring the relationship between northern corn leaf blight and yield losses. Plant Dis. 65:325-327.

25. Raymundo, A. D., and Hooker, A. L. 1982. Single and combined effects of monogenic and polygenic resistance on certain components of northern leaf blight development. Phytopathology 72:99-103.

26. Raymundo, A. D., Hooker, A. L., and Perkins, J. M. 1981. Effect of gene $H t N$ on the development of northern corn leaf blight epidemics. Plant Dis. 65:327-330.

27. Robert, A. L., and Findley, W. R. 1952. Diseased corn leaves as a source of infection and natural epidemics of Helminthosporium turcicum. Plant Dis. Rep. 36:9-10.

28. Schechert, A. L., Welz, H. G., and Geiger, H. H. 1999. QTL for resistance to Setosphaeria turcica in tropical African maize. Crop Sci. 39:514-523.

29. Sigulas, K. M., Hill, R. R., Jr., and Ayers, J. E. 1988. Genetic analysis of Exserohilum turcicum lesion expansion on corn. Phytopathology 78:149-153.

30. Smith, D. R., and Kinsey, J. G. 1980. Further physiologic specialization in Helminthosporium turcicum. Plant Dis. 64:779-781.

31. Smith, D. R., and Kinsey, J. G. 1993. Latent period - a possible selection tool for Exserohilum turcicum resistance in corn (Zea mays L.) Maydica 38:205-208.

32. Smith, D. R., and White, D. G. 1988. Diseases of corn. Corn and Corn Improvement. G. F. 
Sprague and J. W. Dudley, eds. Agronomy 18:687-766.

33. Thakur, R. P., Leonard, K. J., and Jones, R. K. 1989. Characterization of a new race of Exserohilum turcicum virulent on corn with resistance gene $H t N$. Plant Dis. 73:151-155.

34. Turner, M. T., and Johnson, E. R. 1980. Race of Helminthosporium turcicum not controlled by $\mathrm{Ht}$ genetic resistance in corn in the American Corn Belt. Plant Dis. 64:216-217.

35. Ullstrup, A. J. 1963. Sources of resistance to northern corn leaf blight. Plant Dis. Rep. 47:107-108.

36. Ullstrup, A. J. 1970. A comparison of monogenic and polygenic resistance to Helmin- thosporium turcicum in corn. Phytopathology 60:1597-1599.

37. Ullstrup, A. J., and Miles, S. R. 1957. The effects of some leaf blights of corn on grain yield. Phytopathology 47:331-336.

38. Welz, H. G., and Geiger, H. H. 2000. Genes for resistance to northern leaf blight in diverse maize populations. Plant Breed. 119:1-14.

39. Welz, H. G., Wagner, R., and Geiger, H. H. 1993. Virulence variation in Setosphaeria turcica populations collected from maize in China, Mexico, Uganda, and Zambia. (Abstr.) Phytopathology 83:1356.

40. Welz, H. G., Xia, X. C., Bassetti, P., Melchinger, A. E., and Lubberstedt, T. 1999.
QTLs for resistance to Setosphaeria turcica in an early maturing dent $\times$ flint maize population. Theor. Appl. Genet. 99:649-655.

41. White, D. G., ed. 1999. Compendium of Corn Diseases. 3rd ed. American Phytopathologica Society, St. Paul, MN

42. Wilcoxson, R. D., Atif, A. H., and Skovmand, B. 1974. Slow rusting of wheat varieties in the field correlated with stem rust severity on detached leaves in the greenhouse. Plant Dis. Rep. 58:1085-1087.

43. Windes, J. M., and Pedersen, W. L. 1991. An isolate of Exserohilum turcicum virulent on maize inbreds with resistance gene $H t N$. Plant Dis. 75:430. 